\title{
Immunohistochemical Staining for Lymphatic and Blood Vessels in Normal Tissues: Comparison between Routinely Paraffin-Embedded Tissues and Frozen Sections
}

\author{
Tatsuo Tomita \\ Departments of Integrative Biosciences and Pathology, Oregon Health and Science University, Portland, OR, USA \\ Correspondence: tomitat39@gmail.com; Tel.: + 1971470 7252; Fax: + 15034948311
}

Received: 18 January 2021; Accepted: 17 February 2021

\begin{abstract}
Objective. In the current study, we compared the distribution of blood and lymphatic vessels from paraffin-embedded tissues with those of frozen tissues of normal human and rhesus monkey. Materials and Methods. We performed immunocytochemical staining for lymphatic and blood vessels using LYVE-1 for lymphatic vessels and von Willebrand factor (F-8) for blood vessels. Results. Normal tissues included spleen, lymph node, liver, pancreas, salivary gland, colon, diaphragm, heart, lung, thyroid, adrenal gland, kidney, ovary, endometrium, and prostate. Splenic sinusoids were stained for LYVE-1 and F-8 in the frozen sections, supporting that the sinusoid is a lymphoreticular system and blood vessel in structure and function. In frozen sections, the lymphatic sinusoids were consistently positive for LYVE-1, while hepatic sinusoids were positive for LYVE-1, but not for F-8. Thus, lymphatic and blood vessels were more readily detected in frozen tissue sections than in the paraffin-embedded sections. In the endometrium, lymphatic vessels were not diffusely immunostained in paraffin-embedded sections. However, frozen sections detected cyclic changes of lymphatic vessels, growing from basalis to functionalis in the menstrual cycle. Lymphatic vessels were immunostained in many organs using frozen sections. Small pulmonary blood vessels were not immunostained by F-8 in the periphery of the bronchial vessel tree most likely these smallest blood vessels were not immunostained due to less F- 8 attached to their endothelia. Conclusion. The present findings illustrate the differences in the immunostaining of blood vessels in sections obtained from paraffin-embedded tissues and those from frozen tissue. These new findings may be relevant for the basic histology and histopathology of lymphatic and blood vessels.
\end{abstract}

Key Words: Blood Vessels • Lymphatic Vessels • Immunohistochemistry • Factor-8 • LYVE-1.

\section{Introduction}

Practically every organ is supplied by lymphatic vessels except the brain, spinal cord, cartilage, bone marrow, eye lens, and others (1-4). However, the presence of lymphatic vessels in each organ is not definitively identified at the histologic levels. Using immunocytochemical staining for lymphatic and blood vessels, we had tried to detect lymphatic and blood vessels using currently available immunocytochemical markers. The currently commercially available markers for lymphatic vessel endothelia include proxy-1 (prospero-related homeobox-1), LYVE-1 (lymphatic vessel endothelial hyaluronic acid factor receptor-1), podoplanin (43 kDa membrane glycoprotein of podocytes), and VEGFR-3 (vascular endothelial growth factor receptor-3) (1-4). LYVE-1 is a transmembrane receptor for hyaluronan, a highly expressed by lymphatic vessels (3-6). A podoplanin is a membrane glycoprotein found on the surface of rat glomerular epithelial cells, podocytes, recognized by the monoclonal antibody, D2-40 (3). These markers bind to their own specific binding sites in different modes, and they all function in diverse ways at different stages of tissue growth and development (2-4). The markers for blood vessels include CD31 (platelet endothelial adhesion molecule PECASM-1, found on endothelial cells), CD34 (single-class 
transmembrane sialomucin protein, its antibody is used for hematopoietic progenitor cells, positive for blood vessel endothelium but not lymphatic vessel endothelium), and von Willebrand factor (binds factor-8, F-8, a clotting factor in blood vessel endothelium, platelet aggregation and adhesion to the cell wall of injured vessels), which are pan-vascular endothelial markers (3).

However, there are truly no specific markers for lymphatic vessels and blood vessels, respectively, at present $(2,3,7-10)$. We had previously performed such immunocytochemical staining with normal tissues. We found that frozen sections were superior for lymphatic and blood vessels immunostaining than the routinely formalin-fixed and paraffin-embedded sections $(11,12)$. To anatomic pathologists, it is crucially important to identify the precise presence of lymphatic and blood vessels in the normal organs and the pathological tissue specimens, especially in cancer tissues $(11,12)$. The classical staining for lymphatic vessels is van Gieson's stain for the lining elastic lamina for distinguishing lymphatic vessels from the small tissue spaces due to the fixation artifacts by buffered formalin-fixation and paraffin embedding. The presence of red blood cells in the endothelium lining vessel lumens supports to identify blood vessels. These two vessel systems supply the organs for lymph fluid and blood and have crucial roles in cancer spread and metastasis.

The aim of the current study was to compare the distribution of blood and lymphatic vessels using paraffin-embedded and frozen tissue samples of normal human and rhesus monkey. We employed mostly LYVE-1 for lymphatic vessels and von Willebrand factor (Factor-8, F-8) for blood vessels stains (13).

\section{Materials and Methods}

Normal human tissues were collected from surgically removed organs at the Department of Pathol- ogy, University of Kansas Medical Center, Kansas City. These tissues included colon and pancreas. Immunocytochemical staining procedures with paraffin-embedded tissues were previously reported $(11,12)$. The sources of antibodies used for immunocytochemical staining and each dilution of the antibody for routinely formalin-fixed and paraffin-embedded tissues and frozen sections are as follows (Table 1).

For frozen sections, normal organ tissues from Macaca mulatta (rhesus monkey) were procured by necropsy at the laboratory of Drs. Robert Brenner and Ov Slayden, Oregon National Research Center, Beaverton, OR. The frozen tissues were prepared from the liver, spleen, lymph node, diaphragm, heart, lung, thyroid, adrenal gland, kidney, ovary, uterus, and prostate. Additionally, monkey liver and spleen were fixed in a mixture of $1 \%$ paraformaldehyde and $1 \%$ formalin and were embedded in paraffin. With spleen and liver, double immunocytochemical staining was performed with paraffin sections for LYVE-1 and F-8 using brown color by diaminobenzidine tetrahydrochloride and blue color by Vectastain and Vector SG (Burlingame, CA). For frozen sections, small fresh tissues $(1 \times 1 \times 0.4 \mathrm{~cm})$ were embedded in OCT matrix (Fisher Scientific, Pittsburgh, PA) and were frozen in liquid propane in the liquid nitrogen bath as described before (13-18) and were frozen sectioned at 5-7 microns. Frozen sections were mounted on Super Frost Plus slides (Fisher Scientific), microwaved-irradiated on ice for $3 \mathrm{sec}$, fixed in $2 \%$ paraformaldehyde in phosphate buffer at $\mathrm{pH}$ 7.4 for 10 to $15 \mathrm{~min}$ at room temperature, and immersed twice for $2 \mathrm{~min}$ in $85 \%$ ethanol (13). To inhibit endogenous peroxidase activity, sections were incubated with a solution containing glucose oxidase $(1 \mathrm{U} / \mathrm{ml})$ and sodium azide $(10 \mathrm{mmol} /$ $\mathrm{ml}$ ) in PBS for $45 \mathrm{~min}$ at $25^{\circ} \mathrm{C}$ (13). Sections were incubated with blocking serum for $20 \mathrm{~min}$. Then,

Table 1. Antibodies that Were Used in the Current Study

\begin{tabular}{llll}
\hline Antibody (clone) & Manufacturer & Paraffin sections dilution & Frozen sections dilution \\
\hline Goat-anti human LYVE-1 & R \& D System, Minneapolis, MN & $1: 100$ & $1: 1.200$ \\
\hline Mouse monoclonal D2-40 & Signet Laboratories, Dedham, Mass & $1: 100$ & $1: 100$ \\
\hline Rabbit anti-human F-8 & Dako System, Carpenteria, CA & $1: 100$ & $1: 800$ \\
\hline
\end{tabular}


sections were incubated with each diluted primary antibody solution overnight at $4^{\circ} \mathrm{C}$. After rinsing and immersion in blocking serum again, sections were incubated with a second antibody (1: $200 \mathrm{di}-$ lution) for $30 \mathrm{~min}$ at room temperature. Final visualization was achieved with the ABC kit (Vector Laboratories, Burlingame, CA) and 0.025 diaminobenzidine tetrahydrochloride in Tris-buffer $\mathrm{pH}$ 7.6, $0.03 \% \mathrm{H}_{2} \mathrm{O}_{2}$ to induce brown color.

\section{Results}

\section{Spleen and Lymph Node (Figure 1)}

Frozen sections of the spleen showed diffuse staining in the sinusoidal endothelia and no staining in the central arterioles in the germinal center for LYVE-1, while F-8 staining revealed positive staining in the central arterioles and adjacent larger arteries (Figure 1-A and -B). The positive F-8 staining in the sinusoidal epithelia was only achieved in the frozen section, not in the routinely processed paraffin sections (Figure 1-B and -D). Double staining for LYVE-1 in brown and F-8 in blue (Figure 1-C) and F-8 in brown and LYVE-1 in blue (Figure 1-D), both revealed positive staining in the sinusoidal endothelia for LYVE-1 and central arterioles staining for F-8. There was no F-8 staining sinusoids in the paraffin sections (Figure 1-D). Thus, the red pulp's sinusoidal epithelia were double-positive for LYVE-1 and F-8 only in the frozen section (Figure 1-A and -B). The frozen sections of the lymph node showed a few lymphatic vessels in the cortex. Simultaneously, there were numerous slender or round lymphatic vessels in the medulla and the connective tissue of the hilum (Figure 1-E). There were lymphatic sinusoidal
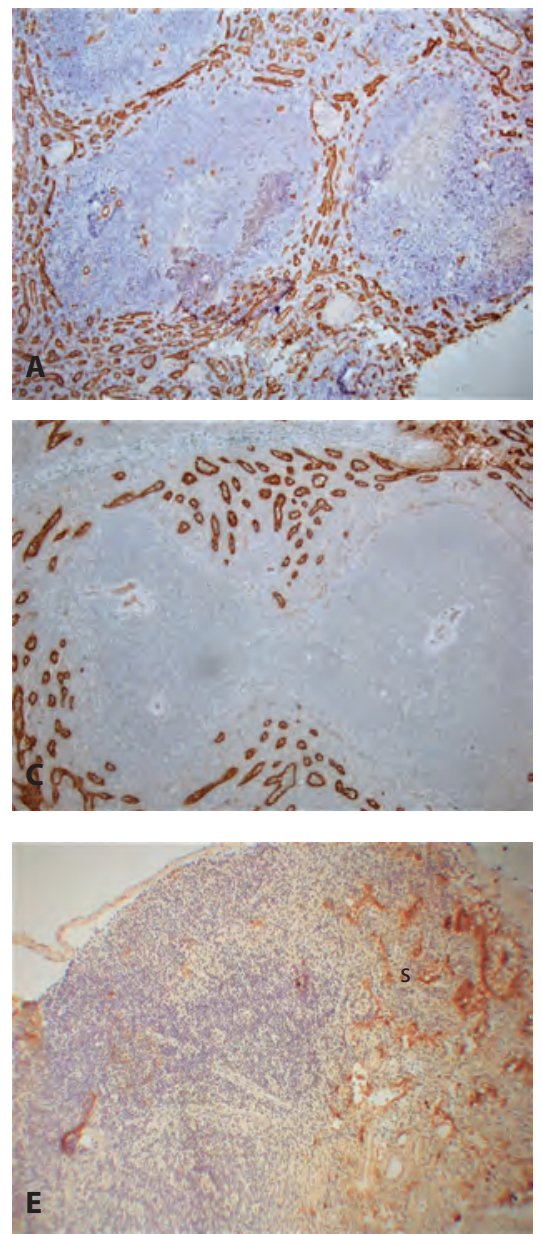
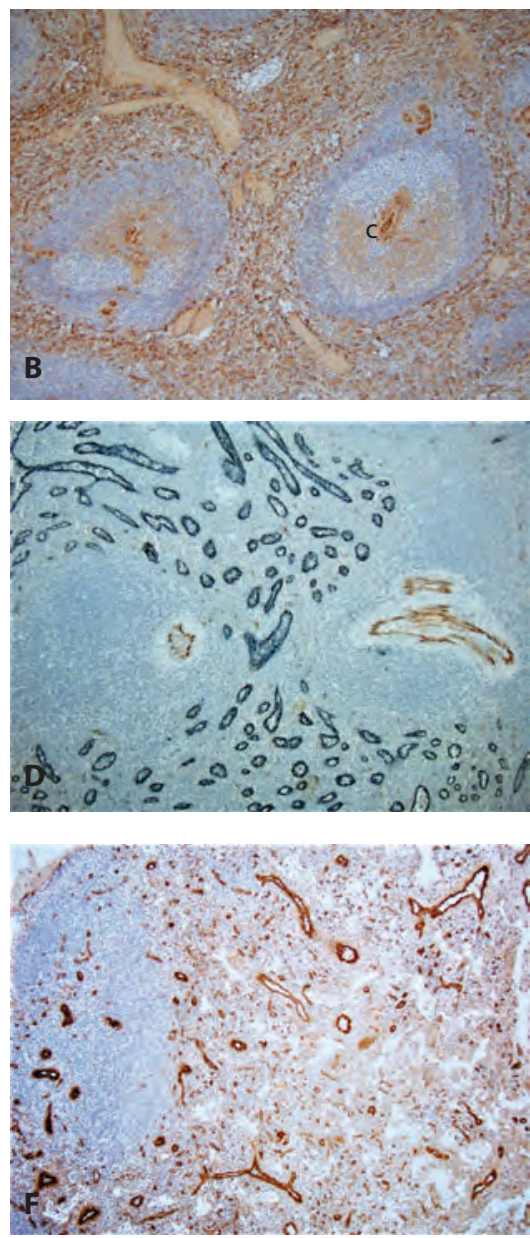

Figure 1. Spleen and Lymph Node. The frozen sections of spleen showed diffuse positive staining in the sinusoids for both LYV-1 and F-8 (A and B), and central arterioles and larger blood vessels were strongly positive for F-8 (B). The paraffin-embedded sections were double stained for LYVE-1 in brown and $\mathrm{F}-8$ in blue, showing positive in sinusoids for LYVE-1 and positive in blood vessels for F-8 (C), and in brown for blood vessels and in blue for sinusoids (D). There was no positive staining for $\mathrm{F}-8$ in the splenic sinusoid in the paraffin-embedded section (D). The frozen sections of lymph node showed LYVE-1 positive staining in lymphatic sinusoids at the subcapsular-medullary junction and linear lymphatic vessels in medulla and hilar connective tissue (E ), where abundant F-8 positive blood vessels were present $(F)$. $A$, $B, E$ and $F=$ Frozen sections; $C$ and $\mathrm{D}=$ Paraffin-embedded sections, $\mathrm{c}$ : Central artery, s: Lymphatic sinus; $A$ and $E=L Y V E-1 ; B$ and $F=F-8$, immunostained; $C=$ LYVE-1/F-8; $D=F-8 /$ LYVE-1 double-immunostained. 

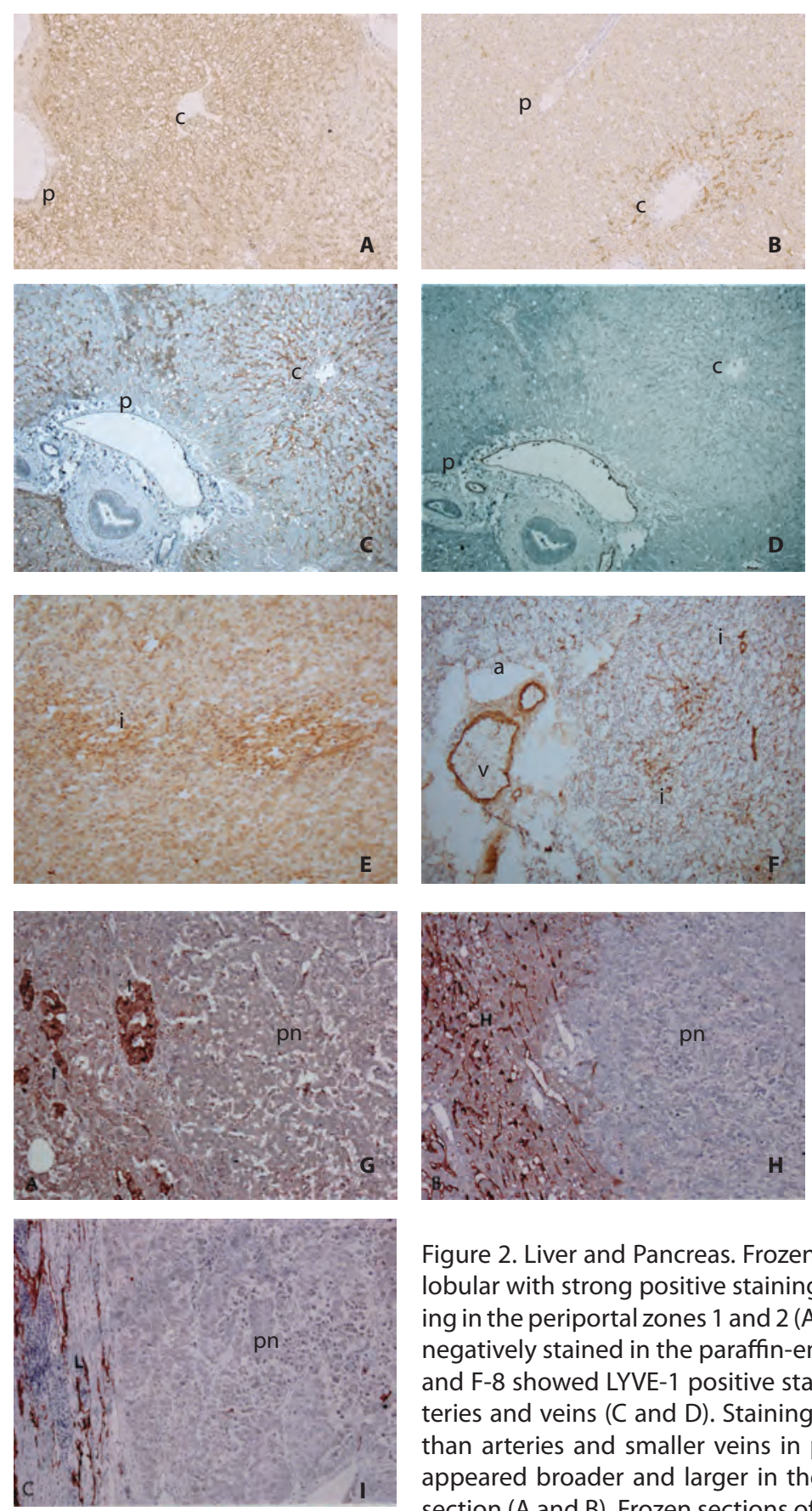

epithelia in the subcapsular (marginal)medullar junction of the lymph node, strongly positive only for LYVE-1 (Figure 1-E). Mostly round thick-walled arteries were less positively stained for F-8 than thin-walled, dilated veins, which were stronger stained for F-8 with abundant endothelial staining in the small blood vessels in the medulla and large blood vessels in the hilum (Figure 1-F).

\section{Liver and Pancreas (Figure 2)}

For LYVE-1 immunostaining, the staining between frozen section and paraffin-embedded section was compared, showing darker staining in the relatively broader liver sheets in the frozen section (Figure 2-A) than the weaker staining in the relatively thinner liver sheets in paraffin-embedded section (Figure 2-B). In the frozen section, pan-lobular sinusoids were diffusely positive for LYVE-1 with stronger pericentral sinusoids than periportal sinusoids. Some periportal sinusoids were barely positive or negative (Figure 2-A). In contrast, the periportal sinusoids were not stained for LYVE-1 in the paraffin-embedded section (Figure 2-B). For F-8 staining, blood

Figure 2. Liver and Pancreas. Frozen section for LYVE-1 staining showed diffuse panlobular with strong positive staining in the pericentral zone 3 and less positive staining in the periportal zones 1 and $2(A)$ while periportal zone 1 and most of zone 2 were negatively stained in the paraffin-embedded sections (B). Double staining for LYVE-1 and F-8 showed LYVE-1 positive staining in sinusoids and F-8 positive staining in arteries and veins ( $C$ and $D)$. Staining for $F-8$ revealed stronger stained in larger veins than arteries and smaller veins in paraffin-embedded section (D). Liver cell sheets appeared broader and larger in the frozen section than in the paraffin-embedded section ( $A$ and $B)$. Frozen sections of pancreas showed barely weakly stained islets by LYVE-1 (E) and F-8 staining showed islets consisting of round baskets of abundant, entangled F-8 positive capillaries (F). Paraffin-sections of pancreas showed strong staining in islets and there was no staining for primary non-functioning Pan$\operatorname{NET}(\mathrm{G})$, metastatic insulinoma in liver $(\mathrm{H})$ and metastatic gastrinoma in lymph node (I). Lymphatic vessels in the pancreatic stroma (G), hepatic sinusoid $(\mathrm{H})$ and lymphatic sinus (I) were positively immunostained for LYVE-1 (G-I), a: Artery, c: Central vein, i: Islet, I: Lymphatic vessel, p: Portal area, pn: Pan-NET, v: Vein. A, E, F=Frozen section; B, C, D, G-I=Paraffin-embedded sections; A, B, E, G-I=LYVE-1, ; C=LYVE-1/F-8 double stained; $D=F-8 / L Y V E-1$ double stained; $F=F-8$ immunostained. Figures $\mathrm{G}, \mathrm{H}$ and $\mathrm{I}$ are from the reference 33 (Tomita T. Pancreas. 2007;35(4):e18-22). 
vessels were strongly stained in larger vessels while sinusoids were negative in the paraffin-embedded section (Figure 2-D). Double immunostaining for LYVE-1 and F-8 showed sinusoidal staining endothelial staining in brown for LYVE-1 and weak F-8 staining in arteries and veins in blue (Figure 2-C) and vice versa for brown staining for F-8 and blue staining for LYVE-1 (Figure 2-D).

Frozen section stained for LYVE-1 revealed weakly stained pancreatic islets (Figure 2-E). In contrast, F-8 staining revealed numerous pancreatic islets, consisting of a round basket of abundant, tangled capillaries, and thick-walled arteries and thin-walled veins were moderately and strongly stained, respectively (Figure 2-F). Paraffin-embedded tissues stained strongly for LYVE-1 in pancreatic islets with adjacent lymphatic vessels but no LYVE-1 staining in 23 out of 25 cases of pancreatic neuroendocrine tumors (Pan-NETs) (Figure 2-G), including metastatic insulinoma to the liver (Figure 2-H) and metastatic gastrinoma to lymph node (Figure 2-I). Hepatic and lymphatic sinusoids were positively stained for LYVE-1 in paraffin-embedded sections (Figure 2-H and -I).

\section{Colon, Diaphragm, and Salivary Gland (Figure 3)}

In the paraffin-embedded colonic sections, there were numerous slender lymphatic vessels in the submucosa, including lymphoid follicle with a few lymphatic vessels in lamina propria (Figure
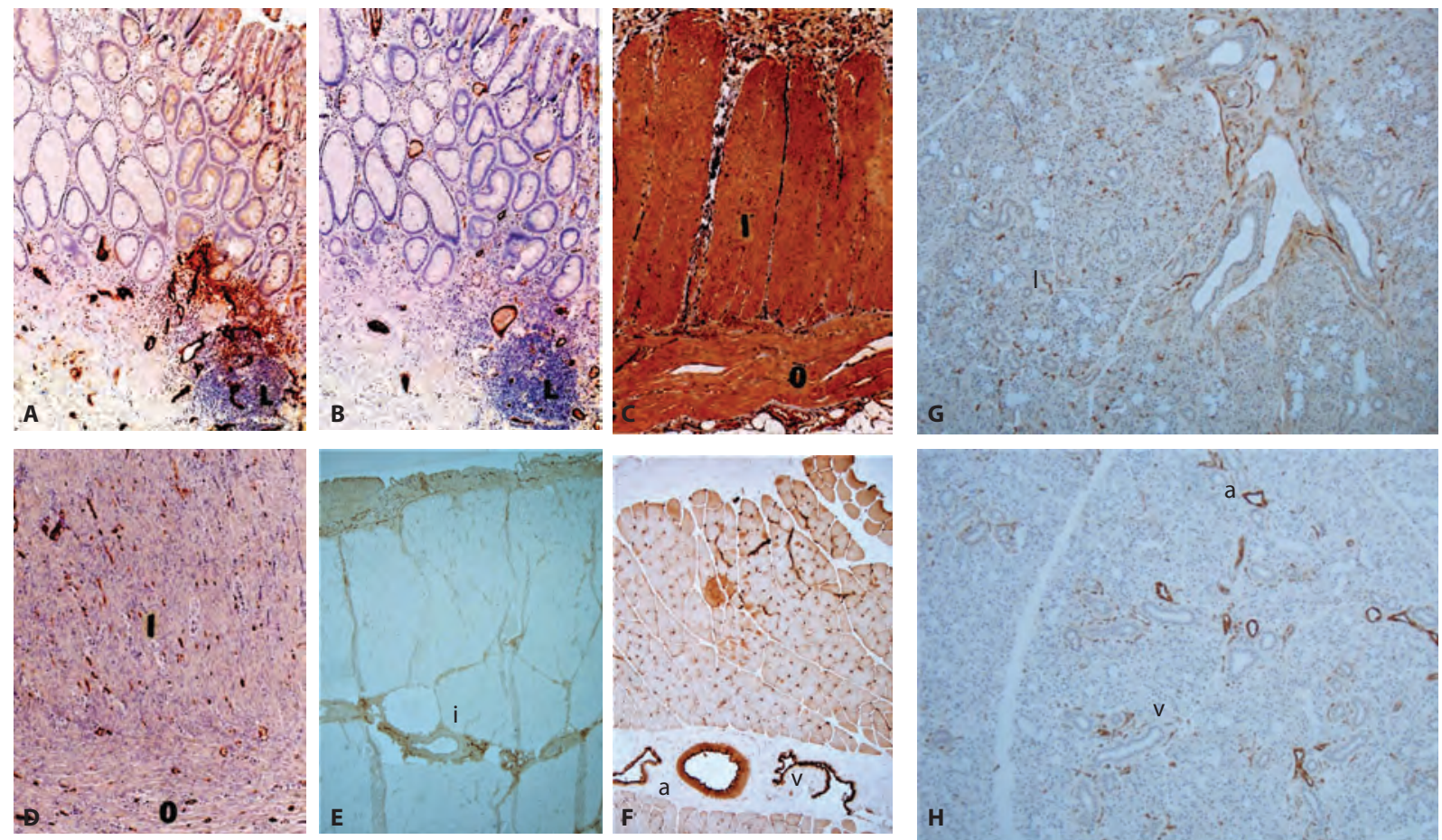

Figure 3. Colon, Diaphragm and Salivary Gland. Paraffin-embedded sections of colon showed a few lymphatic vessels by LYVE-1 staining and numerous round blood vessels by F-8 staining in lamina propria while submucosa contained numerous linear lymphatic vessels and many round blood vessels including the lymphoid follicle (A and B). Lymphatic and blood vessels longitudinally crisscrossed the inner circular and outer longitudinal muscle layer (C and D). Frozen sections of diaphragm contained linear and many small lymphatic vessels in the arterial adventitia of fibrous septa (E) and small capillaries at the outer margins of the striated muscle with many blood vessels in the fibrous septa (F). Frozen sections of salivary gland showed abundant, scattered small linear lymphatic vessels $(G)$ and numerous scattered round blood vessels $(H)$, a: Artery, i: Inner circular muscle, I: Lymphatic vessel, o: Outer longitudinal muscle, v: Vein. A-D=Paraffin-embedded sections; $\mathrm{E}-\mathrm{H}=$ Frozen sections; $\mathrm{A}, \mathrm{C}$ and $\mathrm{G}=\mathrm{LYVE}-1 ; \mathrm{B}, \mathrm{D}, \mathrm{F}, \mathrm{H}=\mathrm{F}-8$; $\mathrm{E}=\mathrm{D} 2-40$ immunostained; Figures A-D are from the reference 12. Tomita T. Cancer-associated lymphatic and venous vessels in colonic carcinomas. Open J Pathol. 2014;4(2):101-9. 
3-A). There were abundant round, dilated veins in the mucosa and submucosa, with some inside the lymphoid follicle (Figure 3-B). Many lymphatic and blood vessels penetrated longitudinally through the two layers of smooth muscles (Figure 3-C and -D). The diaphragm's frozen section was transversely supplied with a scanty, linear network of lymphatic vessels in the thin, fibrous septum and arterial adventitia (Figure 3-E). The striated muscles were diffusely and richly supplied by capillaries at the outer margins of the muscle bundles. On the other hand, abundant thick-walled arteries and thin-walled veins were stained in the endothelia for F-8, with veins stronger stained than arteries (Figure 3-F). The frozen section of the salivary gland revealed many scattered small lymphatic vessels in the periductal and interlobular stroma (Figure 3-G). There were numerous scattered F-8 positive small blood vessels in the inter-lobular stroma, including thick-walled arteries and thinwalled veins in the periductal stroma (Figure 3-H).

\section{Thyroid, Adrenal Gland, and Kidney (Figure 4)}

In the thyroid, there were abundant linear lymphatic vessels in interfollicular septa (Figure 4-A). In contrast, more abundant larger thick-walled arteries and thin-walled, plump veins were strongly positive for F-8 in the thyroid septa with veins stronger stained than arteries in the frozen sections (Figure 4-B). The adrenal gland contained numerous small lymphatic vessels and larger, dilated blood vessels in the subcapsular tissue (Figure 4-C and -D). There were few lymphatic vessels in the cortical and medullary septa (Figure $4-C$ and -D). In the kidney, there were only a few small linear lymphatic vessels around the glomeruli and very few lymphatic vessels in the cortical stroma (Figure 5-E). Glomerular capillaries were densely and diffusely stained for F-8 with positive staining spreading outside the capillary, suggestive of the leaking endothelia (Figure 5-F). Both thick-walled arteries and thinwalled veins were moderately and strongly stained for F-8, respectively (Figure 5-F).
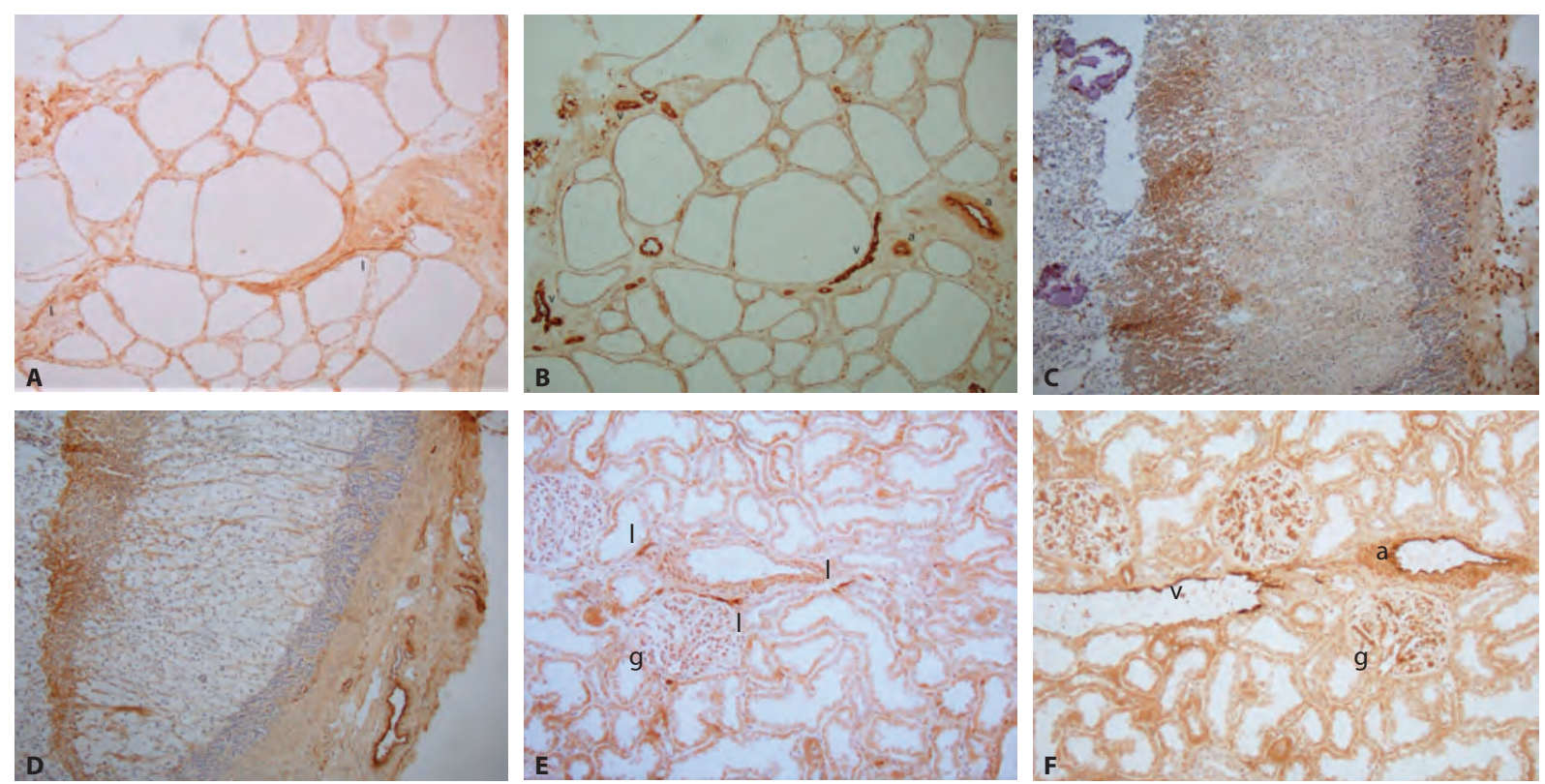

Figure 4. Thyroid, Adrenal Gland and Kidney. Frozen sections of thyroid showed linear and many small lymphatic vessels in fine fibrous septa (A) and more abundant larger, round blood vessels in the septa (B). Adrenal sub-capsular stroma contained numerous small, linear lymphatic vessels (C) and larger round blood vessels (D). There were few lymphatic vessels in the cortex and medulla in kidney. Frozen sections of kidney showed a few small, liner lymphatic vessels around the glomerulus and tubules (E) while glomerular capillaries were densely and diffusely stained for F-8 spreading outside of the capillary, suggestive of leaking blood vessels. There was strong staining for F-8 in larger veins than in arteries (F), a: Artery, g: Glomerulus, I: Lymphatic vessel, v: Vein; A-F=Frozen sections; A, C, E=LYVE-1; B, D, F=F-8 immunostained. 


\section{Heart, Lung, Ovary, and Prostate (Figure 5)}

Numerous, small, scattered lymphatic vessels were diffusely present in the full thickness of the cardiac ventricle, and much less and a few blood vessels were present in the sections of the ventricle with larger vessels in epicardium than in endocardium (Figure 5A and -B). In the lung, lymphatic vessels were diffusely scattered in the wall of terminal bronchioles, respiratory bronchioles, and alveolar ducts (Figure 5-C). In contrast, fewer blood vessels were revealed by F-8 staining in the wall of mostly terminal bronchioles and respiratory bronchioles (Figure 5-D). Numerous linear lymphatic vessels were present around the ovarian follicle and interstitial stroma, linear with a narrow lumen (Figure 5-E). Likewise, numerous small blood vessels were present around the ovarian follicle in the stroma with plump lumens (Figure 5-F). The prostatic sub-capsule and stroma contained many scattered small, linear lymphatic vessels (Figure 5-G). There were more numerous small blood vessels than lymphatic vessels in the sub-capsule and interlobular stroma than lymphatic vessels (Figure $5-\mathrm{H}$ ). There were many nerve fibers in the prostatic sub-capsule (5-G and $-\mathrm{H})$.

\section{Macaca mulatta Endometrium, Days 3, 14, and 28 of Menstrual Cycle (Figure 6)}

The cyclic endometrium was stained in the frozen sections. The endometrium from the Day 3 of the menstrual cycle showed thin residual basalis, which contained some small lymphatic vessels (Figure 6-A) and numerous dilated blood vessels (Figure 6-B). Day 14 en-
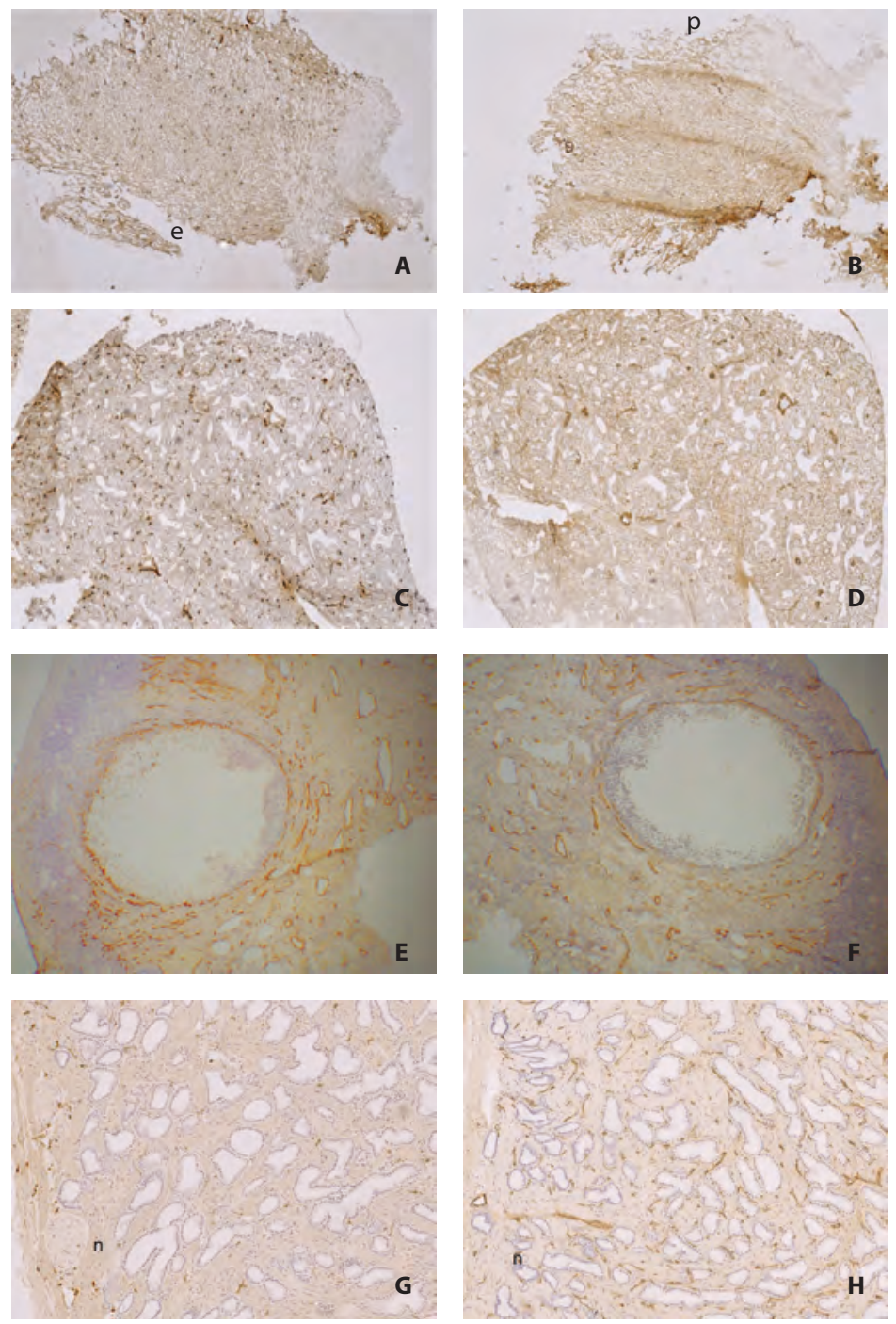

Figure 5. Heart, Lung, Ovary and Prostate. Frozen sections of heart showed numerous scattered small lymphatic vessels (A) and less abundant larger blood vessels with larger caliber in the pericardium (B). There were abundant lymphatic vessels in the wall of terminal bronchioles, respiratory bronchioles, and alveolar ducts (C) while there were less, large blood vessels in in the wall of terminal bronchioles and respiratory bronchioles (D). There was no staining for F-8 in the alveolar capillaries (D). Frozen sections of ovary showed numerous, scattered small lymphatic vessels around the ovarian follicle and interstitial stroma (E), and likewise there were numerous, plump blood vessels in the stroma and around the follicle (F). Prostate showed many small scattered lymphatic vessels in the sub-capsular and interstitial stroma $(G)$ and more numerous small blood vessels in the stroma $(H)$. There were many nerve bundles in the prostatic sub-capsule ( $G$ and $H)$, e: Endocardium, n: Nerve, p: Pericardium. A to $\mathrm{H}=$ Frozen sections; $\mathrm{A}, \mathrm{C}, \mathrm{E}, \mathrm{G}=\mathrm{LYVE}-1 ; \mathrm{B}, \mathrm{D}, \mathrm{G}, \mathrm{H}=\mathrm{F}-8$ immunostained. 
dometrium showed many small lymphatic vessels in the lower stratum functionalis but few lymphatic vessels in the growing upper functionalis (Figure 6-C). Simultaneously, there were numerous blood vessels in both lower and upper functiona-
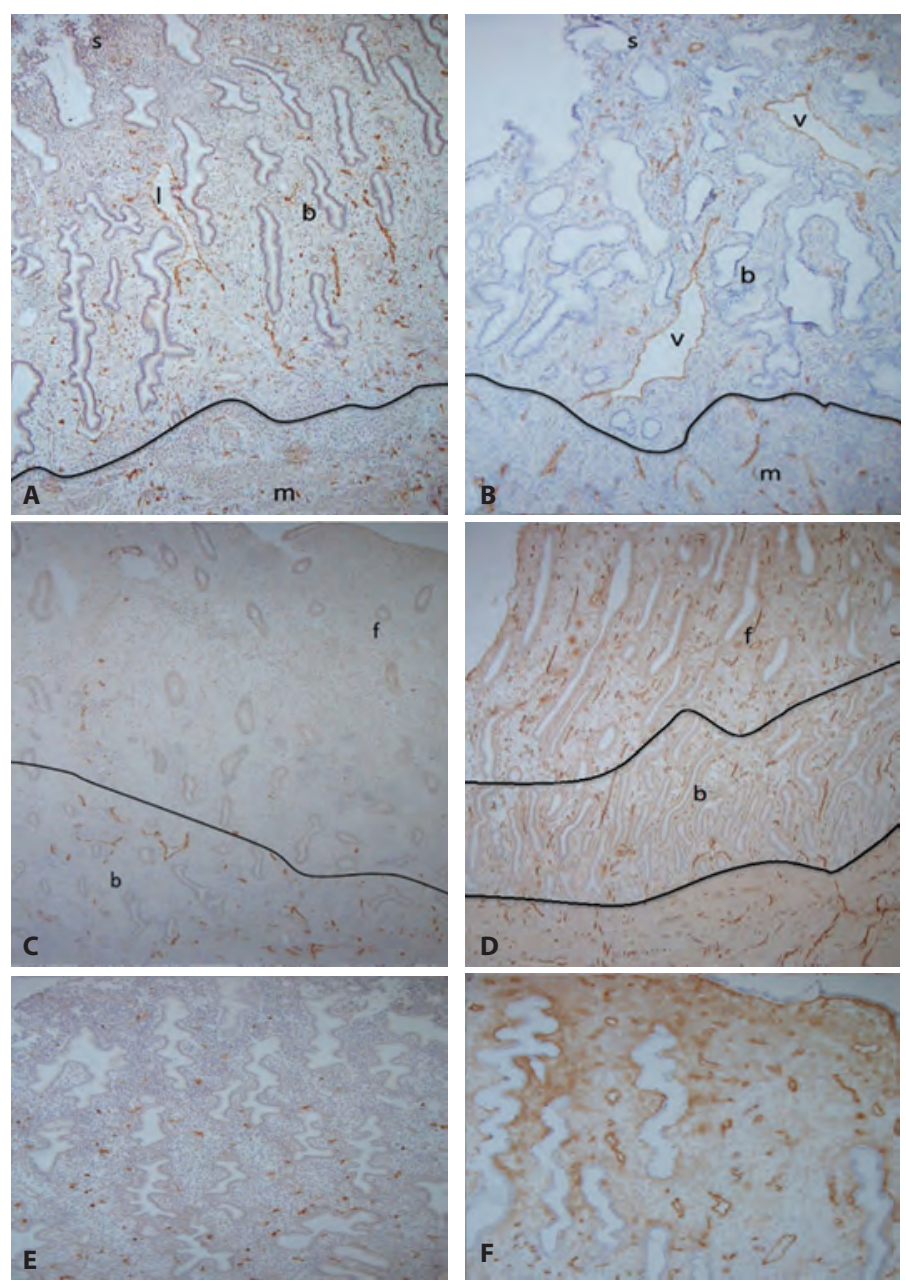

Figure 6. Macaca Mulatta Endometrium, Day 3, 14 and 28 of Menstrual Cycle. Day 3 endometrium of the menstrual cycle showed thin residual basalis, which contained some, small lymphatic vessels (A) and numerous small blood vessels (B). Day 14 endometrium showed a few small, lymphatic vessels (C) and numerous elongating blood vessels in the growing functionalis (D). Day 28 in menstrual cycle, there were several perpendicular small lymphatic vessels in the upper stratum functionalis as compared to numerous small lymphatic vessels in the lower functionalis (E). There were dilated, densely F-8 positive venous vessels, suggestive of leaking blood vessels, which were located perpendicular to the uterine cavity in the upper functionalis (F) as compared to slim elongated blood vessels in the lower functionalis and basalis (D). In myometrium, there were many perpendicular and transverse lymphatic vessels (A, B, D). b: Basalis; f: Functionalis; I: Lymphatic vessel; $m$ : Myometrium; v: Dilated vessel; $A-F=$ Frozen sections; $A, C$, $\mathrm{E}=\mathrm{LYVE}-1 ; \mathrm{B}, \mathrm{D}, \mathrm{F}=\mathrm{F}-8$ immunostained. lis (Figure 6-D). In the endometrium of the 28th day of menstrual cycle, there were small scattered ymphatic vessels in the upper functionalis (Figre 6-E). There were numerous, dilated, densely -8-stained blood vessels spreading outside of the endothelium, suggestive of leaking blood vessels located perpendicular to the uterine cavity in the upper functionalis (Figure 6-F). There were many perpendicular and transverse lymphatic vessels in the myometrium (Figure 6-A, -B, -D). There were even more numerous larger venous vessels in myometrium than in functionalis and basalis (Figure 6-D).

\section{Discussion}

There are many immunologic and immunohistochemical markers for lymphatic and blood vessels but what we need is just one solely reliable and specific marker for lymphatic vessel and blood vessel, respectively. However, there is none for that purpose at present. The structure-function relationship of lymphatic vasculature has been overshadowed and delayed by a lack of specific markers for lymphatic vessels (1-4). As shown in the dilution of the primary antibody, the frozen sections required a tiny fraction of primary antibodies with much more diluted solutions, especially for the primary LYVE-1 antibody; thus, frozen section immunostaining was much more economical than paraffin-embedded sections since these antibodies were expensive. The hind side of frozen sections is that the frozen tissue preparation is quite cumbersome and labor-intensive. Only small tissue $(1 \times 1 \times$ $0.4 \mathrm{~cm}$ ) may be adequately processed in our method (13-18). During the freezing process, the tissue may crack, the larger tissue tends to crack more often than the smaller tissue, and the freezing process needs to be improved for future study. We used frozen sections of 5-7 $\mu \mathrm{m}$ in thick- 
ness, and it was not an easy task to cut thin frozen sections to see a clear view of vascular vessels, and the larger the tissue is, the harder it is to cut good thin sections. Furthermore, frozen sections on the glass slides stored in a deep freezer may degenerate with increasing non-specific staining if kept for more than four weeks. Liver and kidney sections are more prone to degenerate during storage in a deep freezer at $-70 \mathrm{C}^{\circ}$. For the best staining result, the fresh frozen sections are the best for immunocytochemical staining immediately after sectioning. The buffered $10 \%$ formalin fixation decreased organ weight by about $10 \%$, but liver sheets were shrunk more than 10\% (Figure 2-A and -B).

The clearly different staining pattern was revealed in the spleen, where paraffin-embedded sections revealed LYVE-1 positive sinusoidal epithelia, which were large capillaries and were negative for F-8 (Figure 1-D). Thus, the F-8 positive staining in the splenic sinusoidal epithelia was only positively achieved in the frozen sections (Figure 1-B) but not in the paraffin-embedded sections (Figure 1-D). There were different staining patterns between the frozen section and paraffinembedded section for splenic sinusoids. In a study using paraffin-embedded sections, splenic sinus was reportedly only patchy positive for F- 8 and negative for CD34 (4). The splenic red pulp is lined by distinctive endothelial cells with a partial histologic function called splenic littoral cells, which express endothelial markers like F-8, CD 31, WT1 (Wilms' tumor protein 1), ERG (a member of the erythroblast transformation-specific family), and CD68 (a protein highly expressed by the cells in the monocyte lineage in circulating blood and tissue macrophages) (1-4). Blood is sequestrated in the splenic sinus in the red pulp under the condition of portal hypertension. The sinusoid of larger caliber allows transporting whole blood cells between the capillary wall and the adjacent tissue. Its endothelia increase the attachment of F- 8 on the splenic littoral cell surface resulting in strong F-8 staining on the surface (Figure 1-B). Since splenic sinusoidal epithelia are positive for both F-8 and LYVE-1, these epithelia are both venous and lymphatic endothelia, as seen in the frozen sections.
They may function as both venous capillaries and lymphatic capillaries. The human spleen's sinusoid is an unusual vessel, which is involved in the removal of damaged erythrocytes and permits the migration of leukocytes from the cords into the circulation (4). This endothelium of the sinusoids is equivalent to other endothelium in their immunoreactivity to F-8 and HLA-DR antigens (4, 19-21). The white splenic pulp, germinal center, consists of central arterioles (Figure 1 A-D), which are surrounded by lymphoid cells, the socalled periarteriolar sheath (PALS), and adjacent outpouchings of nodular lymphoid tissue $(3,4$, 19-21). The lymph node sections also showed a different staining pattern for lymphoid sinusoids, positive for LYVE-1 in the frozen sections. Using paraffin-embedded sections, the lymphoid sinus was reportedly positive for CD34 and negative for CD31 (4). The lymphoid sinusoids are located between the subcortical (marginal)-medullary junction (Figure 1-E). Lymph nodes filter protein-rich lymph fluid through lymphatic sinusoids, while the spleen filters blood through splenic sinusoids $(3,4)$. In lymph nodes, there were abundant arteries and veins in the medullary cord and hilum with small lymphatic vessels in the periarterial stroma (Figure 1-E and -F). Double staining for LYVE-1 and F-8 was possible only with paraffin-embedded sections, and an attempt for double staining with frozen sections was unsuccessful because of too much non-specific background staining, which resulted in too dirty to identify vessels at our hand.

LYVE-1 immunostaining is not restricted to the lymphatic vessels but is expressed in normal hepatic sinusoids, which are downregulated in hepatic cancer and liver cirrhosis (22). The hepatic sinusoids were positively stained for LYVE-1, not entirely in all zones in paraffin-embedded section where oxygen-rich zones 1 (periportal hepatocytes) was negative but oxygen-poor zones 2 and 3 (pericentral hepatocytes) were positive (Figure $2-\mathrm{A}$ and $-\mathrm{B}$ ). However, all zones 1 to 3 in some tissue areas were positively stained for LYVE1 in the frozen section. So, zone 3 was stronger stained than zones 1 and 2 (Figure 2-A). There are two types of sinusoidal epithelia in the hepa- 
tocytes. Type 1 sinusoidal epithelia are LYVE-1, $\mathrm{CD} 32^{\text {hi }} \mathrm{CD} 14^{+}$hepatocyte in the oxygen-rich zone 1 , and type 2 sinusoidal epithelia are LYVE- $1^{+}$, $\mathrm{CD} 32^{\text {hi }} \mathrm{CD} 14^{+} \mathrm{CD} 36^{\text {mid-lo }}$ in the oxygen-poor zone 2 and 3 hepatocytes (Figure 2-A and -B) (22-26). Thus, LYVE-1 staining is mostly stronger stained in the zones 2 and 3 endothelia, which are venous capillaries with more LYVE-1 attached in the endothelia, while zone 1 is arterial capillary with less F-8 attached. Hepatic sinusoids are large capillaries positive for LYVE-1 in zone 3 in the paraffinembedded section, but not diffusely for F-8 with patchy staining in the literature $(3,4,24,25)$. F-8 immunostaining yielded negatively stained in liver sinusoids, while stronger positive staining was seen in veins with larger caliber vessels (Figure 2-C and -D). In paraffin-embedded sections, zone 1 was positive, and zone 2 was negative for CD34 since CD34 stained stronger for arterioles than venules, supporting that zone 1 is arterial capillary $(4,23)$. The frozen section for F-8 staining did not yield positive for hepatic sinusoids $(25,26)$ (Figure not shown). Comparing the sizes of liver sheets, the paraffin-embedded section was smaller than that of frozen sections by more than 10\% (Figure 2-A and -B). Veins with larger calibers were stronger stained than those with smaller calibers, and veins were stronger stained than the arteries of the same caliber. This finding may support that larger veins have more F- 8 attached on the subendothelial surface than that of smaller veins, and veins with slower blood flow may have more F-8 attached on the endothelia than that of arteries with faster blood flow. Since all undamaged endothelia of veins and arteries are positively stained for F-8 (26), F-8 is not necessarily attached to the damaged vascular endothelia but are attached to the undamaged endothelia as well.

In the three sinusoids containing organs (spleen, lymph nodes, and liver), extramedullary hematopoiesis may occur during fetal development and in hematopoietic malignancies (e.g. chronic myelogenous leukemia and myelofibrosis) (4, 27-30). Extramedullary hematopoiesis is a pathological process in which the differentiation of hematopoietic stem/progenitor cells occurs out- side the bone marrow, namely in the sinusoids of spleen, liver and lymph nodes. In the spleen, mesenchymal progenitor-like cells expressing TlX1, an essential transcription factor for spleen organogenesis, are selectively localized in the perifollicular region of the red pulp and are a major source of hematopoietic stem cell factors (31). Thus, many markers of lymphatic and blood vessels may also show diverse immunohistochemical expressions depending on the developmental and hematopoietic neoplastic stages $(2-4,32)$. Hepatic sinusoidal endothelia separate passenger leukocytes in the sinusoidal lumen from hepatocytes and act as a platform for the adhesion of various liver-resident immune cell populations. Thus, the endothelial cells function as scavengers and pose potent immune function as sentinel cells to detect microbial infection $(4,25)$. The pancreas showed strikingly different staining patterns between the two staining procedures: frozen sections barely showed LYVE1 positive pancreatic islets (Figure 2-E) while paraffin sections stained LYVE-1 in islets diffusely and strongly (Figure 2-G). In paraffin-embedded sections, 23 out of 25 cases of Pan-NETs, including non-functioning Pan-NET, metastatic insulinoma in the liver and metastatic gastrinoma in the lymph node, were negative for LYVE-1. Simultaneously, the normal tissues, including pancreatic islets with adjacent lymphatic vessels, hepatic sinusoids, and lymphatic sinusoids, were all positively stained for LYVE-1 (Figure 2-G, -H and -I) $(4,33)$. In contrast, eight out of 25 Pan-NETs were positively stained for D2-40 (Figure not shown) (34). This strong immunostaining was also observed for staining pancreatic hormones in the paraffinembedded sections but not in the frozen sections (Figures not shown). LYVE-1 and D2-40 positive staining in islets support that islets are LYVE-1/ D2-40 filled endocrine organs, with which islets function as a paracrine endocrine organ in the lymphatic medium since all four pancreatic hormones are interacting with each other by either stimulating or inhibiting the secretion of the other hormones for glucose homeostasis of the whole body. In the paracrine islet system, insulin inhibits glucagon secretion, glucagon stimulates insulin 


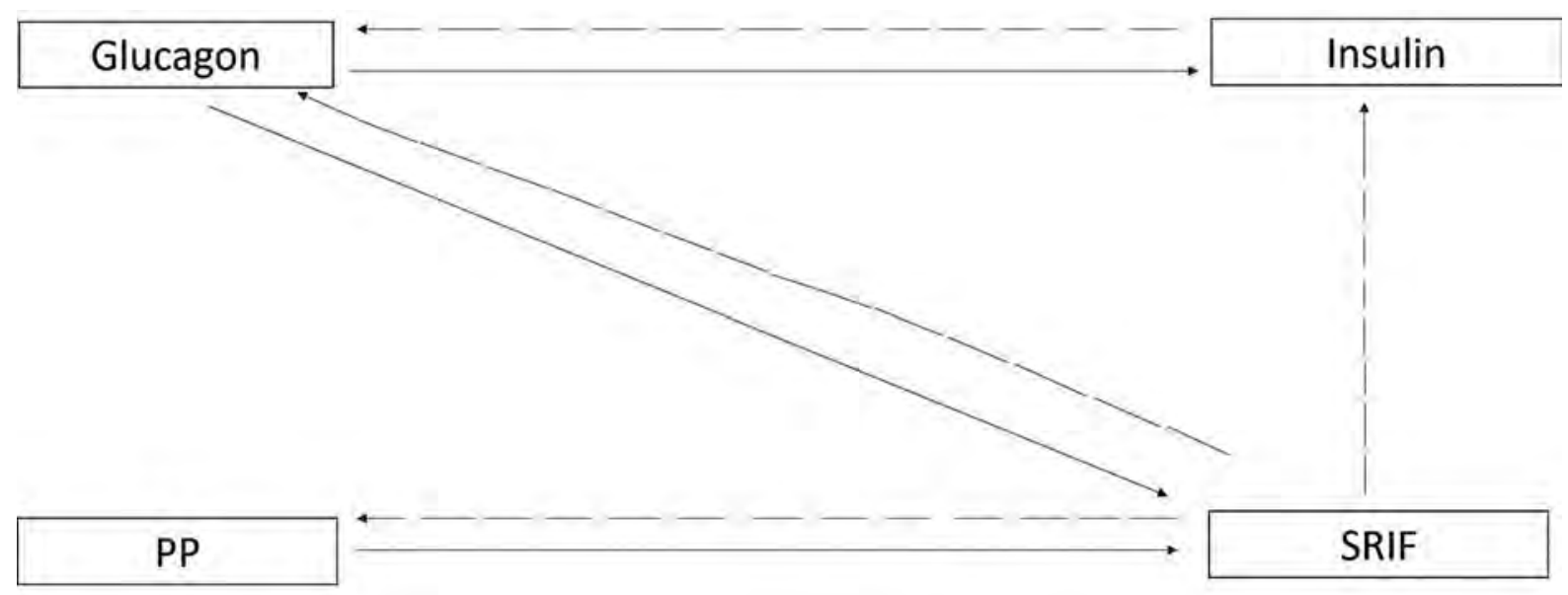

Figure 7. Paracrine System of Four Pancreatic Endocrine Hormones. The solid line with an arrowhead signifies stimulatory effect on the other hormone (s), while the broken line with an arrowhead signifies inhibition on the other hormone (s). For instance, insulin secretion inhibits glucagon secretion while glucagon secretion stimulates insulin secretion. Somatostatin (SRIF) secretion inhibits secretion of the three other hormones secretion and PP secretion inhibits SRIF secretion. From the reference 35 (Tomita, T, Pathol Int. 2002,52(7):425-32).

secretion, somatostatin inhibits insulin, glucagon and pancreatic polypeptide (PP) section, and PP inhibits somatostatin secretion (Figure 7) (35). F-8 immunostaining with frozen sections showed baskets of F-8 positive rich, round capillaries in the islets, showing the presence of numerous, fenestrated capillaries in the endocrine pancreas by this staining (Figure 2-F). Pancreatic islets occupy only $1-2 \%$ of the pancreatic tissue volume but receive $10-23 \%$ of the entire pancreas' blood supply, which supply rich capillaries in the islets $(36,37)$.

The intestine was technically difficult to cut good frozen sections, and we used well-fixed paraffin-embedded sections for immunostaining. The colonic sections showed a few lymphatic vessels in lamina propria but showed numerous lymphatic vessels in the submucosa (Figure 3-A). Kennedy et al. reported absent lymphatic vessels in lamina propria of the normal human colon with somelymphatic vessels in most cases with inflammation and neoplasia (12). We believe their negative staining was due to non-optimal preservation of lymphatic vessels in paraffin-embedded colonic tissue. Colon lacks lacteals seen in the small intestine but is well supplied with lymphatic vessels in lamina propria, and the highest densities of lymphatic vessels are found in the submucosa (12). Both lymphatic and blood vessels penetrated longitudinally through the colonic muscle wall to the subserosa (Figure 3-C and -D) (11), through which colonic cancer cells may spread to the pericolonic lymph nodes $(38,39)$. Blood vessels were abundant in lamina propria and submucosal stroma (Figure 3-B) (12). We observed more non-specific background staining in the routinely paraffin-embedded section than in the frozen sections, as shown in the colonic muscular layers (Figure 3-C and D) (12). Lymphangiogenesis occurs in adult tissue during inflammation, wound healing, and tumorigenesis (2-4). Colonic carcinoma invades through the longitudinal lymphatic vessels through the smooth muscle wall to pericolonic lymph nodes, and some lymphatic vessels are newly formed peritumorous lymphatic vessels $(12,38,39)$. The diaphragm prepared longitudinally cut, and the sections showed a few longitudinal LYVE-1 positive lymphatic vessels in the fibrous septum. Simultaneously, there were numerous small capillaries in the outer margin of the striated muscle bundle (Figure 3-E and -F). This rich blood vessel network is thought to be responsible for the hematogenous spread of cancers, especially lung cancers to adrenal glands, which accounts for $40 \%$ of metastasis (40). The route of lung cancer metastasizes to the adrenal gland is debated, mainly lymphatic route in the early stage of cancer and mainly hematogenous 
spread in the late stage (40). When lung cancer metastasizes through the diaphragm, the hematogenous spread is more likely through the more abundant blood vessels than the less abundant lymphatic vessels (Figure 4-E and -F). Besides, adrenal metastasis is usually in the medulla, not in the adrenal cortex, and is often bilateral. Salivary glands have the highest rate of lymphatic flow per weight of tissues of all regions in the gastrointestinal tract (41) as supported by numerous lymphatic vessels in the gland (Figure 3-G).

The fibrous septum in the thyroid gland contained linear lymphatic vessels and larger round blood vessels (Figure 4-A and -B). These numerous lymphatic vessels and more numerous blood vessels are supported by a classic dye injection study in the dog, in which numerous lymphatic vessels were detected in the fibrous septa (42). The adrenal sub-capsule contains numerous small lymphatic and blood vessels, but few lymphatic vessels in the cortical and medullary parenchymal tissue are described by Merklin (43) (Figure 4-C and $-D)$. The adrenal medulla contains numerous large blood vessels, which emerge from the hilum before forming suprarenal veins and are the site of lung cancer metastasis, bilateral in the rule. When lung cancer metastasizes to the adrenal gland, the cancer cells may reach the medulla via circulating blood through the hilum's blood vessels (44). Kidney and liver tissues showed the most non-specific staining in the frozen sections. The kidney tissue showed a strikingly different distribution of lymphatic vessels and blood vessels: a few lymphatic vessels around the glomeruli and between the tubules (Figure 4-E) (45). Glomerular endothelia were diffusely and densely stained for F-8, suggestive of leaky capillary, while larger blood vessels were stronger stained (Figure 4-F). Thus, we stained fenestrated, leaking glomerular endothelia with F-8 in the frozen sections (Figure 4-F). However, Pusztaszeri et al. reported completely negative staining for F-8 in the glomerular endothelia in the paraffin-embedded sections but were positive for CD31 and CD34 (4). This is one of the major immunostaining differences for F-8 between frozen sections and paraffin-embedded sections.
Ishikawa et al. studied formalin-fixed and paraffin-embedded human kidneys fixed and found abundant D2-40 positive lymphatic vessels in the stroma around the interlobular and arcuate arteries few sporadic lymphatic vessels in those around glomeruli and cortical stroma (45). Lymphatic vessels were seldom present in the medulla (45).

The structure and function of cardiac lymphatic vessels are not known. However, the heart's lymphatic vessels consist of terminal lymphatics, of various diameters and lymphatic plexus that drain continuously subendocardial, myocardial and subepicardial areas (46) and regulate lymph transport healing of the infarcted heart (46). There were numerous small, scattered lymphatic vessels and less numerous blood vessels in the ventricular wall in the frozen sections containing pericardial blood vessels (Figure 5-A and -B). Pusztaszeri et al. described capillaries positively stained for CD31 and CD34 with some staining for F-8, but there was no mention of lymphatic vessels in the heart (4). Kambouchner and Bernaudin studied pulmonary lymphatic vessels in the paraffin-embedded human lung using D2-40 as a marker and detected small lymphatic channels within the normal pulmonary lobules, emerging from intralobular stroma, and around small blood vessels constituting the para-alveolar lymphatic vessels (47). With frozen sections, we found numerous lymphatic vessels in the wall of terminal bronchioles, respiratory bronchioles, to alveolar ducts. In contrast, lesser blood vessels were noted in the terminal bronchioles to respiratory bronchioles (Figure 5-C and -D). Adenocarcinomas of the lung arise at the terminal bronchial tree, have easy access to the rich peribronchial lymphatics, and drain into the hilar lymph nodes $(48,49)$. Alveolar capillaries and arterioles of the lung were reportedly strongly positive for CD31 and CD34 but negative for F-8 (50), reflecting smaller amount of F-8 attached on the subendothelial surface. Small- to large-sized blood vessels were positively stained for F-8 (4). Bronchial arteries supply bronchial trees down to the pulmonary alveolus, however, arterial and venous vessels were not immunostained by F-8 in the periphery of the respiratory bronchioles, likely 
due to less amount of F-8 attached in the small vessel and capillary endothelium.

Ovarian sections showed numerous small lymphatic and blood vessels around the Graafian follicle and in the stroma with rich lymphatic vessels and blood vessels (Figure 5-E and -F). Generally, lymphatic vessels were slender while blood vessels were plump with dilated, round lumens (Figure 5 -E and -F). The ovaries' lymphatic drainage pathways run via the ovarian and uterine ligaments and ovarian cancer may spread through lymphatic vessels to sentinel nodes, para-aortic and para-internal iliac arteries nodes (51). The prostate showed numerous small lymphatic vessels in the subcapsular and interstitial septa, where there were even more numerous, small blood vessels than lymphatic vessels (Figure $5-\mathrm{G}$ and $-\mathrm{H}$ ). There were rich nerve fibers in the sub-capsule (Figure $5-\mathrm{G}$ and $-\mathrm{H})$. By double staining for blood vessels and lymphatic vessels, there were numerous CD34 positive blood vessels but only a few LYVE-1 positive lymphatic vessels in the tissues with benign prostatic hyperplasia and prostatic carcinoma, in which the destruction of lymphatic vessels and angiogenesis occurs (52). Peritumoral lymphatic vessels are likely to serve as major conduits for nodal metastasis (53), which spread to the deep branches of internal iliac lymphatic nodes (54). Rich vasculature in the prostate provides access to hematogenous spread of high-grade prostatic cancer, which is found in 35\% of autopsy cases, frequently involving bones (90\%), lung (46\%), and liver (25\%) (55).

To study lymphatic vessels in the endometrium is a special technical challenge to pathologists since the definite presence of lymphatic vessels had not been reported using paraffin-embedded tissues: some reported no lymphatic vessels in human endometrium (56-58). Red-Horse et al. used 4\% paraformaldehyde-fixed and paraffin-embedded section using LYVE-1 as a lymphatic vessel marker and found no lymphatics in the non-pregnant human uterus (56). Koukourakis et al. studied formalin-fixed and paraffin-embedded human uterus using LYVE-1 and CD31 and found LYVE-1 positive lymphatic vessels in myometrium only (57).
Rogers et al. used paraffin-embedded sections of the human endometrium using D2-40, CD31, and F-8 for immunostaining. They reported lymphatic vessels representing $13 \%$ of all vessels in functionalis, $43 \%$ of basalis, and $28 \%$ in the myometrium, respectively, with no difference in staining intensity between functionalis and basalis lymphatic vessels (58). Rogers et al. concluded that lymphatic vessels in functionalis were significantly reduced compared to basalis across the menstrual cycle (58-61). Their photomicrographs showed weakly immunostained lymphatic vessels in the small, limited tissue areas using D2-40 and VEGF-C as lymphatic vessel markers (58-62), which were not to the same degree of staining reported by us using frozen sections $(63,64)$. These photomicrographs appeared to be taken from the much limited, selectively cherry-picked, positively stained tissue area since there were no large, overview photos of lymphatic vessels at a lower magnification (58-62). Indeed, we had a severe limitation to immunostain lymphatic and blood vessels with routinely processed paraffin-embedded uterine tissues using currently available commercial antibodies. We need better antibodies against these vessels usable for paraffin-embedded tissues of larger than $1 \mathrm{~cm}^{2}$. The suggestive leaking blood vessels in glomerular capillaries and Day 28 endometrium blood vessels were densely immunostained for F-8 spreading outside the blood vessels. These leaking blood vessels were not observed in the paraffin-embedded sections (58-61). Rogers et al. prompted us to pursue the current study despite the elusiveness of lymphatics presence in the endometrium (58$60)$. Arterial changes with the menstrual cycle had been well established. However, lymphatic vessels' possible presence remains an enigma, although lymphatic fluid constitutes a few percent of menstrual fluid $(58,62)$. Using LYVE-1 as a lymphatic vessel marker, we proved that there were also cyclic changes of lymphatic vessels in the endometrium in the menstrual cycle where there were few or no lymphatic vessels in the early proliferative phase functionalis. In contrast, lymphatic vessels proliferated in the later secretory phase, growing from basalis to functionalis, which dilated and burst in 
accord with blood vessel bursting during menstruation $(63,64)$. Thus, the frozen section immunocytochemical staining has validated this method's superiority over paraffin-embedded sections for studying lymphatic and blood vessels. The new findings from frozen sections will eventually shed light on the basic histology and histopathology.

Acknowledgment: I want to express my sincere thanks to Drs. Robert M Brenner and Ov D Slayden who kindly allowed me to use normal organs of rhesus monkey at their research laboratory of the Oregon National Primate Research Center, Beaverton, OR, USA. Their advices and critical comments are highly appreciated, and they prompted me to pursue this study.

Conflict of Interest: The author declares that he has no conflict of interest.

\section{References}

1. Kong LL, Yang NZ, Shi LH, Zhao GH, Zhou W, Ding Q, et al. The optimum marker for the detection of lymphatic vessels. Mol Clin Oncol. 2017;7(4):515-20.

2. Müller AM, Hermanns MI, Skrzynski C, Nesslinger M, Müller KM, Kirkpatrick CJ. Expression of the endothelial markers PECAM-1, vWf, and CD34 in vivo and in vitro. Exp Mol Pathol. 2002;72(3):221-9.

3. Scavelli C, Weber E, Aglianò M, Cirulli T, Nico B, Vacca A, et al. Lymphatics at the crossroads of angiogenesis and lymphangiogenesis. J Anat. 2004;204(6):433-49.

4. Pusztaszeri MP, Seelentag W, Bosman FT. Immunohistochemical expression of endothelial markers CD31, CD34, von Willebrand factor, and Fli-1 in normal human tissues. J Histochem Cytochem. 2006;54(4):385-95.

5. Jackson DG, Prevo R, Clasper S, Banerji S. LYVE-1, the lymphatic system and tumor lymphangiogenesis. Trends Immunol. 2001;22(6):317-21.

6. Jackson DG. The lymphatics revisited: new perspectives from the hyaluronan receptor LYVE-1. Trends Cardiovasc Med. 2003;13(1):1-7.

7. Tammela T, Alitalo K. Lymphangiogenesis: Molecular mechanisms and future promise. Cell. 2010;140(4):460-76.

8. Zheng W, Aspelund A, Alitalo K. Lymphangiogenic factors, mechanisms, and applications. J Clin Invest. 2014; 124(3):878-87.

9. Christiansen A, Detmar M. Lymphangiogenesis and cancer. Genes Cancer. 2011;2(12):1146-58.

10. Stacker SA, Williams SP, Karnezis T, Shayan R, Fox SB, Achen MG. Lymphangiogenesis and lymphatic vessel remodelling in cancer. Nat Rev Cancer. 2014;14(3):159-72.
11. Tomita T. Immunocytochemical localization of lymphatic and venous vessels in colonic polyps and adenomas. Dig Dis Sci. 2008;53(7):1880-5.

12. Tomita T. Cancer-associated lymphatic and venous vessels in colonic carcinomas. Open J Pathol. 2014;4(2):101-9.

13. Slayden OD, Koji T, Brenner RM. Microwave stabilization enhances immunocytochemical detection of estrogen receptor in frozen endothelial cells and lymphatic vessels to grow and invade. Cancer Res. 1995;136(6):4012-21.

14. Critchley HO, Kelly RW, Baird DT, Brenner RM. Regulation of human endometrial function: mechanisms relevant to uterine bleeding. Reprod Biol Endocrinol. 2006;4 Suppl 1(Suppl 1):S5

15. Jabbour HN, Kelly RW, Fraser HM, Critchley HO. Endocrine regulation of menstruation. Endocr Rev. 2006;27(1):17-46.

16. Brenner RM, Slayden OD, Rodgers WH, Critchley HO, Carroll R, Nie XJ, et al. Immunocytochemical assessment of mitotic activity with an antibody to phosphorylated histone $\mathrm{H} 3$ in the macaque and human endometrium. Hum Reprod. 2003;18(6):1185-93.

17. Slayden OD, Brenner RM. A critical period of progesterone withdrawal precedes menstruation in macaques. Reprod Biol Endocrinol. 2006;4 Suppl 1(Suppl 1):S6.

18. Cao W, Mah K, Carroll RS, Slayden OD, Brenner RM. Progesterone withdrawal upregulates fibronectin and integrins during menstruation and repair in the rhesus macaque endometrium. Hum Reprod. 2007;22(12):3223-31.

19. Giorno R. Unusual structure of human splenic sinusoids revealed by monoclonal antibodies. Histochemistry. 1984;81(5):505-7.

20. Jønsson V. Comparison and definition of spleen and lymph node: a phylogenetic analysis. J Theor Biol. 1985;117(4):691-9.

21. Borch WR, Aguilera NS, Brissette MD, O’Malley DP, Auerbach A. Practical Applications in Immunohistochemistry: An Immunophenotypic Approach to the Spleen. Arch Pathol Lab Med. 2019;143(9):1093-105.

22. Mouta Carreira C, Nasser SM, di Tomaso E, Padera TP, Boucher Y, Tomarev SI, et al. LYVE-1 is not restricted to the lymph vessels: expression in normal liver blood sinusoids and down-regulation in human liver cancer and cirrhosis. Cancer Res. 2001;61(22):8079-84.

23. Strauss O, Phillips A, Roggiero K, Barlett A. Immunofluorescence identifies distinct subsets of endothelial cells in the human liver. Sci Rep. 2017;17:44356.

24. Do H, Healey JF, Waller EK, Lollar P. Expression of factor VIII by murine liver sinusoidal endothelial cells. J Biol Chem. 1999;274(28):19587-92.

25. Knolle PA, Wohlleber D. Immunological functions of liver sinusoidal endothelial cells. Cell Mol Immunol. 2016;13(3):347-53.

26. Hollestelle MJ, Thinnes T, Crain K, Stiko A, Kruijt JK, van Berkel TJ, et al. Tissue distribution of factor VIII 
gene expression in vivo--a closer look. Thromb Haemost. 2001;86(3):855-61.

27. Tsamandas AC, Jain AB, Raikow RB, Demetris AJ, Nalesnik MA, Randhawa PS. Extramedullary hematopoiesis in the allograft liver. Mod Pathol. 1995;8(6):671-4.

28. Kim CH. Homeostatic and pathogenic extramedullary hematopoiesis. J Blood Med. 2010;1:13-9.

29. Yamamoto K, Miwa Y, Abe-Suzuki S, Abe S, Kirimura S, Onishi I, et al. Extramedullary hematopoiesis: Elucidating the function of the hematopoietic stem cell niche (Review). Mol Med Rep. 2016;13(1):587-91.

30. Tefferi A. Primary myelofibrosis: 2013 update on diagnosis, risk-stratification, and management. Am J Hematol. 2013;88(2):141-50.

31. Oda A, Tezuka T, Ueno Y, Hosoda S, Amemiya Y, Notsu $\mathrm{C}$, et al. Niche-induced extramedullary hematopoiesis in the spleen is regulated by the transcription factor Tlx1. Sci Rep. 2018;8(1):8308.

32. Negrini D, Moriondo A. Lymphatic anatomy and biomechanics. J Physiol. 2011;589(Pt 12):2927-34.

33. Tomita T. Lymphatic vessel endothelial hyaluronan receptor 1 immunocytochemical staining for pancreatic islets and pancreatic endocrine tumors. Pancreas. 2007;35(4):e18-22.

34. Tomita T. D2-40 immunocytochemical staining for pancreatic islets and pancreatic endocrine tumors. Pancreas. 2009;38(3):339-40.

35. Tomita T. New markers for pancreatic islets and islet cell tumors. Pathol Int. 2002;52(7):425-32.

36. Jansson L, Barbu A, Bodin B, Drott CJ, Espes D, Gao X, et al. Pancreatic islet blood flow and its measurement. Ups J Med Sci. 2016;121(2):81-95.

37. Diez JA, Arrojo E Drigo R, Zheng X, Stelmashenko OV, Chua M, Rodriguez-Diaz R, et al. Pancreatic Islet Blood Flow Dynamics in Primates. Cell Rep. 2017;20(6):1490501.

38. Kenney BC, Jain D. Identification of lymphatics within the colonic lamina propria in inflammation and neoplasia using the monoclonal antibody D2-40. Yale J Biol Med. 2008;81(3):103-13.

39. Alexander JS, Ganta VC, Jordan PA, Witte MH. Gastrointestinal lymphatics in health and disease. Pathophysiology. 2010;17(4):315-35.

40. Milovanovic IS, Stjepanovic M, Mitrovic D. Distribution patterns of the metastases of the lung carcinoma in relation to histological type of the primary tumor: An autopsy study. Ann Thorac Med. 2017;12(3):191-8.

41. Gordon SD. Lymphatic system of the thyroid gland. Can Med Assoc J. 1931;25(1):46-8.

42. Aiyama S, Kikuchi K, Takada K, Ikeda R, Sato S, Kuroki J. Immunohistochemical study of the lymphatic vessels in major salivary glands of the rat. Okajimas Folia Anat Jpn. 2011;87(4):177-80.
43. Merklin RJ. Suprarenal gland lymphatic drainage. Am J Anat. 1966;119(3):359-74.

44. Bazhenova L, Newton P, Mason J, Bethel K, Nieva J, Kuhn P. Adrenal metastases in lung cancer: clinical implications of a mathematical model. J Thorac Oncol. 2014;9(4):4426.

45. Ishikawa Y, Akasaka Y, Kiguchi H, Akishima-Fukasawa Y, Hasegawa T, Ito K, et al. The human renal lymphatics under normal and pathological conditions. Histopathology. 2006;49(3):265-73.

46. Ratajska A, Gula G, Flaht-Zabost A, Czarnowska E, Ciszek E, Jankowska-Steifer E, et al. Comparative and developmental anatomy of cardiac lymphatics. Sci World J. 2014:183170.

47. Huang L-H, Lavine KJ, Randolph GJ. Cardiac lymphatic vessels, transport, and healing of the infarcted heart. JACC Basic Transl Sci. 2017;2(4):477-83.

48. Bennett DE, Sasser WF, Ferguson TB. Adenocarcinoma of the lung in men. A clinicopathologic study of 100 cases. Cancer. 1969;23(2):431-9.

49. Mori M, Andersson CK, Graham GJ, Löfdahl CG, Erjefält JS. Increased number and altered phenotype of lymphatic vessels in peripheral lung compartments of patients with COPD. Respir Res. 2013;14(1):65.

50. Kambouchner M, Bernaudin JF. Intralobular pulmonary lymphatic distribution in normal human lung using D240 antipodoplanin immunostaining. J Histochem Cytochem. 2009;57(7):643-8.

51. Kleppe M, Kraima AC, Kruitwagen RF, Van Gorp T, Smit NN, van Munsteren JC, et al. Understanding lymphatic drainage pathways of the ovaries to predict sites for sentinel nodes in ovarian cancer. Int J Gynecol Cancer. 2015;25(8):1405-14.

52. Trojan L, Michel MS, Rensch F, Jackson DG, Alken P, Grobholz R. Lymph and blood vessel architecture in benign and malignant prostatic tissue: lack of lymphangiogenesis in prostate carcinoma assessed with novel lymphatic marker lymphatic vessel endothelial hyaluronan receptor (LYVE-1). J Urol. 2004;172(1):103-7.

53. Zeng Y, Opeskin K, Horvath LG, Sutherland RL, Williams ED. Lymphatic vessel density and lymph node metastasis in prostate cancer. Prostate. 2005;65(3):222-30.

54. Swanson GP, Hubbard JK. A better understanding of lymphatic drainage of the prostate with modern imaging and surgical techniques. Clin Genitourin Cancer. 2013;11(4):431-40.

55. Bubendorf L, Schöpfer A, Wagner U, Sauter G, Moch $\mathrm{H}$, Willi $\mathrm{N}$, et al. Metastatic patterns of prostate cancer: an autopsy study of 1,589 patients. Hum Pathol. 2000;31(5):578-83.

56. Red-Horse K, Rivera J, Schanz A, Zhou Y, Winn V, Kapidzic M, et al. Cytotrophoblast induction of arterial apoptosis and lymphangiogenesis in an in vivo model of human placentation. J Clin Invest. 2006;116(10):2643-52. 
57. Koukourakis MI, Giatromanolaki A, Sivridis E, Simopoulos C, Gatter KC, Harris AL, et al. LYVE-1 immunohistochemical assessment of lymphangiogenesis in endometrial and lung cancer. J Clin Pathol. 2005;58(2):202-6.

58. Rogers PA, Donoghue JF, Girling JE. Endometrial lymphangiogensis. Placenta. 2008;29 Suppl A:S48-54.

59. Girling JE, Rogers PA. The endometrial lymphatic vasculature: function and dysfunction. Rev Endocr Metab Disord. 2012;13(4):265-75.

60. Donoghue JF, Lederman FL, Susil BJ, Rogers PA. Lymphangiogenesis of normal endometrium and endometrial adenocarcinoma. Hum Reprod. 2007;22(6):1705-13.
61. Rogers PA, Donoghue JF, Walter LM, Girling JE. Endometrial angiogenesis, vascular maturation, and lymphangiogenesis. Reprod Sci. 2009;16(2):147-51.

62. Critchley HO, Maybin JA. Molecular and cellular causes of abnormal uterine bleeding of endometrial origin. Semin Reprod Med. 2011;29(5):400-9.

63. Tomita T, Mah K. Cyclic changes of lymphatic and venous vessels in human endometrium. Open J Pathol. 2014;4(4):194-205.

64. Tomita, T, Mah, K. Lymphatic vessels in the human endometrium: Are they present or absent? J Clin Exp Pathol 2015;5(4):100241. 